01

\title{
Изотопные эффекты в спектрах комплексов с водородными связями. Ангармонические расчеты изотопологов комплекса $\left[\mathrm{F}(\mathrm{HF})_{2}\right]^{-}$
}

\author{
(C) В.П. Булычев, М.В. Бутурлимова, К.Г. Тохадзе
}

Санкт-Петербургский государственный университет, 199034 Санкт-Петербург, Россия

e-mail: v.bulychev@spbu.ru

Поступила в редакцию 27.03.2020 г.

В окончательной редакции 14.04.2020 г.

Принята к публикации 15.04.2020 г.

Рассчитаны частоты и интенсивности ИК полос поглощения симметричных и асимметричных комплексов с водородной связью $\left[\mathrm{FL}_{1} \mathrm{FL}_{2} \mathrm{~F}\right]^{-}\left(\mathrm{L}_{1}, \mathrm{~L}_{2}=\mathrm{K}-\right.$ мезон Ка, протон $\mathrm{H}$, дейтон D и тритон $\left.\mathrm{T}\right)$. Равновесная конфигурация и поверхности потенциальной энергии и дипольного момента изотопологов $\left[\mathrm{FL}_{1} \mathrm{FL}_{2} \mathrm{~F}\right]^{-}$ вычислялись по методу MP2/6-311++ $\mathrm{G}(3 d f, 3 p d)$ с учетом ошибки наложения базисных наборов мономеров. Расчеты спектральных параметров с учетом ангармонических взаимодействий всех колебаний были выполнены с использованием колебательной теории возмущений второго порядка. Варьирование масс $\mathrm{L}_{1}$ и $\mathrm{L}_{2}$ в широких пределах позволило получить значительные изменения форм нормальных колебаний и величин постоянных ангармонических взаимодействий при изотопном замещении. Определены тенденции в изменениях спектральных параметров как при переходе от одного симметричного изотополога к другому, так и при переходе от симметричных изотопологов к асимметричным. В хорошем согласии с экспериментальной информацией предсказана частота полосы валентного колебания D-F в $[\mathrm{FHFDF}]^{-}$и скорректировано отнесение этой полосы.

Ключевые слова: водородная связь, ангармонические расчеты молекулярных комплексов, изотопные эффекты.

DOI: $10.21883 /$ OS.2020.08.49701.122-20

\section{Введение}

Анионный комплекс $\left[\mathrm{F}(\mathrm{HF})_{2}\right]^{-}$с энергией связи около $300 \mathrm{~kJ} \cdot \mathrm{mol}^{-1}$ является одним из наиболее прочных комплексов с водородной связью. В этом комплексе дополнительный электрон локализуется на центральном атоме фтора и получающийся анион $\mathrm{F}^{-}$участвует в двух линейных водородных связях $\mathrm{F}^{-} \ldots \mathrm{H}-\mathrm{F}$. Этот комплекс является хорошей модельной системой, так как, с одной стороны, многие эффекты водородной связи проявляются в нем очень сильно, а с другой - из-за небольшого количества электронов квантово-механические расчеты этого комплекса могут быть выполнены достаточно точно. В отличие от родственного комплекса $[\mathrm{FHF}]^{-}$ комплекс $\left[\mathrm{F}(\mathrm{HF})_{2}\right]^{-}$изучался экспериментально только в кристаллических соединениях и многокомпонентных растворах. Рентгеноструктурный анализ [1] показал, что комплекс $\left[\mathrm{F}(\mathrm{HF})_{2}\right]^{-}$имеет симметрию точечной группы $C_{2 v}$, угол $\mathrm{F} \ldots \mathrm{F}^{-} \ldots \mathrm{F}$ близок к $135^{\circ}$, расстояние $R\left(\mathrm{~F}^{-} \ldots \mathrm{F}\right)=2.33$ А̊и атомы водорода расположены асимметрично в линейных мостиках $\mathrm{F}$...H-F. Спектры поглощения $\left[\mathrm{F}(\mathrm{HF})_{2}\right]^{-}$и $\left[\mathrm{F}(\mathrm{DF})_{2}\right]^{-}$в области $450-2500 \mathrm{~cm}^{-1}$ были зарегистрированы в кристаллах $\mathrm{KH}_{2} \mathrm{~F}_{3}$ и $\mathrm{KD}_{2} \mathrm{~F}_{3}$ [2]. Спектры $\left[\mathrm{F}(\mathrm{HF})_{2}\right]^{-}$в области $350-2700 \mathrm{~cm}^{-1}$ были также измерены в кристаллах и растворах, содержащих ионы калия [3] и различ- ные амины [4,5]. Полосы поглощения, обнаруженные в этих работах в области $455-480 \mathrm{~cm}^{-1}$, были приписаны валентным модам $\mathrm{F}^{-} \ldots \mathrm{F}$, полосы в области $1020-1150 \mathrm{~cm}^{-1}$ отнесены к деформационным колебаниям $\mathrm{F}^{-} \ldots \mathrm{H}-\mathrm{F}$, а сильная полоса около $1800 \mathrm{~cm}^{-1}$ была приписана валентному колебанию $\mathrm{H}-\mathrm{F}$ без указания его симметрии. Спектры поглощения анионов $\left[\mathrm{F}(\mathrm{HF})_{2}\right]^{-}, \quad\left[\mathrm{F}(\mathrm{DF})_{2}\right]^{-}$и $[\mathrm{FHFDF}]^{-}$, изолированных в аргоновых и неоновых матрицах при $T=12 \mathrm{~K}$, были зарегистрированы в работе [6]. Полосы при 1815 и $1391 \mathrm{~cm}^{-1}$ в аргоновых матрицах были приписаны антисимметричным колебаниям $\mathrm{H}-\mathrm{F}$ и $\mathrm{D}-\mathrm{F}$ в $\left[\mathrm{F}(\mathrm{HF})_{2}\right]^{-}$ и $\left[\mathrm{F}(\mathrm{DF})_{2}\right]^{-}$соответственно. Полоса при $1707 \mathrm{~cm}^{-1}$ была отнесена к колебанию $\mathrm{H}-\mathrm{F}$ в смешанном комплексе $[\mathrm{FHFDF}]^{-1}$. Авторы [6] высказали предположение, что частоты симметричных валентных колебаний $\mathrm{H}-\mathrm{F}$ и $\mathrm{D}-\mathrm{F}$ в $\left[\mathrm{F}(\mathrm{HF})_{2}\right]^{-}$и $\left[\mathrm{F}(\mathrm{DF})_{2}\right]^{-}$должны быть ниже частот соответствующих антисимметричных колебаний.

Первые квантово-механические расчеты структуры и колебательного спектра поглощения комплекса $\left[\mathrm{F}(\mathrm{HF})_{2}\right]^{-}$были выполнены на довольно низком полуэмпирическом или неэмпирическом уровне с использованием гармонического приближения (например, [7,8]). В наших предыдущих работах [9-13] структурные и спектральные параметры комплекса $\left[\mathrm{F}(\mathrm{HF})_{2}\right]^{-}$и неко- 
торых его изотопологов были рассчитаны на более высоком квантово-химическом уровне и с учетом ангармонических взаимодействий. В [9] равновесная ядерная конфигурация комплексов $\left[\mathrm{F}(\mathrm{LF})_{2}\right]^{-}(\mathrm{L}=\mathrm{H}, \mathrm{D}, \mathrm{T})$ была рассчитана по методу MP2/6-311++G $(3 d f, 3 p d)$ с учетом ошибки наложения базисных наборов мономеров. Для этих комплексов вариационным методом были решены четырехмерные ангармонические задачи в пространстве координат двух валентных колебаний мономеров L-F и двух валентных колебаний водородных связей и определены частоты и интенсивности переходов, связанных с возбуждением этих валентных колебаний. Была доказана ошибочность предположения [6] о том, что полоса поглощения симметричного валентного колебания мономеров L-F имеет меньшую частоту, чем полоса антисимметричного колебания. В [9] были также рассчитаны колебательно усредненные значения межъядерных расстояний и показано, что среднее по основному состоянию значение $R\left(\mathrm{~F}^{-} \ldots \mathrm{F}\right)$ должно понижаться при замещении H/D, т. е. был предсказан обратный эффект Уббелоде. В [10] аналогичные расчеты были выполнены для комплексов $\left[\mathrm{F}(\mathrm{KaF})_{2}\right]^{-}$и $[\mathrm{FKaFTF}]$, в которых протоны заменены положительными К-мезонами (каонами) и тритоном. Целью работы являлось расширение диапазона изменения массы частицы L и изучение взаимодействия валентных колебаний двух мономеров $\mathrm{KaF}$ и TF со значительно различающимися приведенными массами. Проблема взаимодействия колебаний мономеров с различными приведенными массами была более тщательно исследована в [11] при расчете колебаний асимметричных комплексов $[\mathrm{FHFDF}]^{-},[\mathrm{FHFTF}]^{-}$ и $[\mathrm{FDFTF}]^{-}$. На основе четырехмерных ангармонических расчетов было показано, что в асимметричных комплексах $\left[\mathrm{FL}_{1} \mathrm{FL}_{2} \mathrm{~F}\right]^{-}$частота валентного колебания $\mathrm{L}_{i}-\mathrm{F}(i=1$ или 2) близка к среднеарифметическому значению частот симметричного и антисимметричного колебаний в комплексе $\left[\mathrm{F}\left(\mathrm{L}_{i} \mathrm{~F}\right)_{2}\right]^{-}$, и колебание более тяжелого мономера частично сохраняет свойство антисимметричности. В [12] вариационный метод решения ангармонических колебательных задач был применен к исследованию взаимодействия валентных колебаний $\mathrm{H}-\mathrm{F}$ с деформационными колебаниями водородного мостика. Были обнаружены сильные резонансы между фундаментальными переходами по валентным колебаниям $\mathrm{H}-\mathrm{F}$ и обертонами деформационных колебаний Н-мостиков. Следствием этих резонансов является перенос интенсивности от полос поглощения валентных колебаний к обертонным полосам деформационных мод. Недостатком работ [9-12] было то, что ангармонические взаимодействия учитывались только при рассмотрении подсистем колебаний с размерностью не выше четыpex. В [13] структурные параметры и колебательный спектр поглощения комплексов $\left[\mathrm{F}(\mathrm{HF})_{2}\right]^{-}$и $\left[\mathrm{F}(\mathrm{DF})_{2}\right]^{-}$ были рассчитаны путем решения вариационным методом колебательных задач при явном рассмотрении ангармонических взаимодействий в шестимерных подсистемах координат внутренних валентных колебаний мономеров и валентных, и плоских деформационных колебаний водородных мостиков. Были получены более точные, чем в [9], значения $R\left(\mathrm{~F}^{-} \ldots \mathrm{F}\right)$ в $\left[\mathrm{F}(\mathrm{HF})_{2}\right]^{-}$ и $\left[\mathrm{F}(\mathrm{DF})_{2}\right]^{-}$, и была определена причина появления обратного э ффекта Уббелоде. Частоты и интенсивности фундаментальных, обертонных и составных переходов были также рассчитаны по теории возмущений с учетом ангармонических взаимодействий между всеми колебательными степенями свободы. Это позволило уточнить значения частот некоторых колебательных полос поглощения, в частности, удалось получить хорошее согласие между теоретическими и экспериментальными [6] значениями частот антисимметричных колебаний $\mathrm{H}-\mathrm{F}$ и $\mathrm{D}-\mathrm{F}$.

Целью настоящей работы является расчет колебаний комплексов $\left[\mathrm{F}(\mathrm{KaF})_{2}\right]^{-}, \quad\left[\mathrm{F}(\mathrm{TF})_{2}\right]^{-}, \quad[\mathrm{FKaFHF}]^{-}$, $[\mathrm{FKaFDF}]^{-}, \quad[\mathrm{FKaFTF}]^{-}, \quad[\mathrm{FHFDF}]^{-}, \quad[\mathrm{FHFTF}]^{-}$и $[\mathrm{FDFTF}]^{-}$с использованием колебательной теории возмущений. Анализ результатов этого расчета вместе c результатами аналогичного расчета $\left[\mathrm{F}(\mathrm{HF})_{2}\right]^{-}$ и $\left[\mathrm{F}(\mathrm{DF})_{2}\right]^{-}[13]$ позволит найти закономерности изменений форм нормальных колебаний комплексов $\left[\mathrm{FL}_{1} \mathrm{FL}_{2} \mathrm{~F}\right]^{-}$и ангармонических взаимодействий между ними при варьировании масс ядер $\mathrm{L}_{1}$ и $\mathrm{L}_{2}$ в широком диапазоне. Будет определено влияние изотопного замещения на частоты и интенсивности фундаментальных и некоторых обертонных и составных спектральных переходов. Целью данной работы является также более полное, чем в работах $[10,11]$, изучение взаимного влияния водородных мостиков в асимметричных комплексах $\left[\mathrm{FL}_{1} \mathrm{FL}_{2} \mathrm{~F}\right]^{-}$. Будут проанализированы константы ангармонического взаимодействия между нормальными колебаниями, которые оказывают влияние на значения энергий колебательных состояний и на частоты спектральных переходов. Результаты такого анализа могут оказаться полезными при изучении других молекулярных комплексов с разветвленными связями.

\section{Методы расчета}

В настоящей работе, так же как и в работах [9-13], квантово-химические расчеты были выполнены по методу MP2/6-311++G $(3 d f, 3 p d)$ с поправкой на ошибку наложения базисных наборов мономеров с использованием пакета программ Gaussian 16 [14]. В равновесной ядерной конфигурации комплекса $\left[\mathrm{F}(\mathrm{HF})_{2}\right]^{-}$все ядра лежат в одной плоскости, и комплекс обладает симметрией $C_{2 v}$. Межъядерные расстояния $R(\mathrm{HF})$ и $R\left(\mathrm{~F}^{-} \ldots \mathrm{F}\right)$, полученные в этих расчетах, имеют равновесные значения 0.9955 и $2.3659 \AA$, угол $\mathrm{F} \ldots \mathrm{F}^{-} \ldots \mathrm{F}=130^{\circ}$, а энергия образования комплекса равна $295.1 \mathrm{~kJ} \cdot \mathrm{mol}^{-1}$. Эти теоретические значения межъядерных расстояний и угла F. . F $\mathrm{F}^{-}$...F достаточно близки экспериментальным данным [1]. В [13] оптимальная конфигурация комплекса была также определена с использованием более точного метода $\operatorname{CCSD}(\mathrm{T}) / \mathrm{d}$-aug-cc-pVTZ, который 
Таблица 1. Гармонические значения частот $v\left(\mathrm{~cm}^{-1}\right)$ и интенсивностей $S\left(\mathrm{~km} \cdot \mathrm{mol}^{-1}\right)$ ИК полос поглощения изотопологов комплекса $\left[\mathrm{F}(\mathrm{HF})_{2}\right]^{-}$

\begin{tabular}{|c|c|c|c|c|c|c|c|c|c|c|}
\hline \multirow{2}{*}{ Полоса } & \multicolumn{10}{|c|}{$v(S)$} \\
\hline & {$[\mathrm{FKaFKaF}]^{-}$} & {$[\mathrm{FHFHF}]^{-}$} & {$[\mathrm{FDFDF}]^{-}$} & {$[\text {FTFTF }]^{-}$} & {$[\mathrm{FKaFHF}]^{-}$} & {$[\mathrm{FKaFDF}]^{-}$} & {$[\mathrm{FKaFTF}]^{-}$} & {$[\text {FHFDF }]^{-}$} & {$[\text {FHFTF }]^{-}$} & {$[\text {FDFTF }]^{-}$} \\
\hline$v_{1}=v_{\mathrm{FF}}-\mathrm{F}$ & $46(0.03)$ & $46(0.03)$ & $46(0.04)$ & $45(0.05)$ & $46(0.02)$ & $46(0.03)$ & $46(0.04)$ & $46(0.04)$ & $46(0.04)$ & $46(0.04)$ \\
\hline$v_{2}=v_{\mathrm{Hb}, \mathrm{str}, \mathrm{s}}$ & 353 (10) & $349(10)$ & $341(9)$ & $333(9)$ & $351(10)$ & $346(10)$ & $342(12)$ & $344(10)$ & $340(11)$ & $336(9)$ \\
\hline$v_{3}=v_{\mathrm{Hb}, \mathrm{str}, \mathrm{a}}$ & $442(235)$ & $438(237)$ & $428(240)$ & $419(239)$ & $439(237)$ & $435(238)$ & $431(236)$ & $432(240)$ & $428(238)$ & $423(240)$ \\
\hline$v_{4}=v_{\mathrm{Hb}, \mathrm{in}, \mathrm{s}}$ & $1690(299)$ & $1200(163)$ & $864(98)$ & $719(77)$ & $1650(169)$ & $1646(131)$ & $1664(545)$ & $1171(97)$ & $1170(89)$ & $843(68)$ \\
\hline$v_{5}=v_{\mathrm{Hb}, \mathrm{in}, \mathrm{a}}$ & $1610(25)$ & $1140(19)$ & $816(19)$ & $675(24)$ & $1170(78)$ & $840(53)$ & $697(46)$ & $839(50)$ & $697(45)$ & $694(40)$ \\
\hline$v_{6}=v_{\mathrm{Hb}, \text { out }, \mathrm{s}}$ & $1615(391)$ & $1148(207)$ & $826(111)$ & $686(66)$ & $1157(111)$ & $829(62)$ & $686(45)$ & $829(62)$ & $686(46)$ & $686(45)$ \\
\hline$v_{7}=v_{\mathrm{Hb}, \mathrm{out}, \mathrm{a}}$ & $1645(3)$ & $1164(3)$ & $830(1)$ & $683(2)$ & $1628(191)$ & $1628(196)$ & $1628(196)$ & $1154(103)$ & $1154(104)$ & $827(60)$ \\
\hline$v_{8}=v_{\mathrm{L}-\mathrm{F}, \mathrm{s}}$ & $3896(1260)$ & $2787(632)$ & $2026(324)$ & $1700(220)$ & $3715(4052)$ & $3697(4652)$ & $3694(4780)$ & $2658(2009)$ & $2649(2231)$ & $1950(826)$ \\
\hline$\nu_{9}=v_{\mathrm{L}-\mathrm{F}, \mathrm{a}}$ & $3445(8779)$ & $2475(4353)$ & $1813(2173)$ & $1532(1444)$ & $2587(3458)$ & $1901(1639)$ & $1585(677)$ & $1889(1730)$ & $1597(1095)$ & $1583(1253)$ \\
\hline
\end{tabular}

Примечание. Результаты расчетов $[\mathrm{FHFHF}]^{-}$и $[\mathrm{FDFDF}]^{-}$взяты из [13].

привел к следующим значениям $R(\mathrm{HF}), R\left(\mathrm{~F}^{-} \ldots \mathrm{F}\right)$, угла $\mathrm{F} \ldots \mathrm{F}^{-} \ldots \mathrm{F}$ и энергии образования комплекca: $0.9948 \AA, 2.3567 \AA, 131.5^{\circ}$ и $301.5 \mathrm{~kJ} \cdot \mathrm{mol}^{-1}$. Близость значений соответствующих параметров, полученных с использованием двух методов, свидетельствует о достаточной точности приближения МР2/6$311++\mathrm{G}(3 d f, 3 p d)$ для расчета структуры и энергетических параметров изучаемых комплексов. Значения $-0.770,-0.664$ и 0.549 зарядов на центральном атоме $\mathrm{F}$, на каждом из атомов $\mathrm{F}$ мономеров $\mathrm{HF}$ и на каждом атоме $\mathrm{H}$, полученные из анализа естественных орбиталей связей, дают представление о степени ионности центрального атома $\mathrm{F}$ и полярности связей $\mathrm{H}-\mathrm{F}$.

Ангармонические расчеты частот и интенсивностей спектральных переходов для комплексов $\left[\mathrm{F}(\mathrm{KaF})_{2}\right]^{-}$, $\left[\mathrm{F}(\mathrm{TF})_{2}\right]^{-}, \quad[\mathrm{FKaFHF}]^{-}, \quad[\mathrm{FKaFDF}]^{-}, \quad[\mathrm{FKaFTF}]^{-}$,

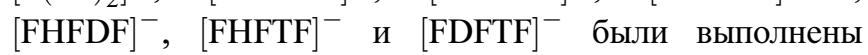
по методу колебательной теории возмущений второго порядка, сформулированному в работах $[15,16]$ и реализованному в программе Gaussian 16 [14]. Достоинством этого метода является введение поправок, получаемых решением вспомогательных вариационных задач, в случае резонансов между близкими по энергии колебательными состояниями. Помимо ангармонических значений частот и интенсивностей, в данной работе анализировались гармонические значения этих спектральных параметров, формы нормальных координат и ангармонические постоянные, входящие в выражения для энергии колебательных состояний.

\section{Расчеты колебаний изотопологов комплекса $\left[\mathrm{F}(\mathrm{HF})_{2}\right]^{-}$в гармоническом приближении}

В табл. 1 приведены значения частот и интенсивностей фундаментальных полос поглощения рассматриваемых комплексов, полученные в гармоническом приближении. Использованы следующие обозначения колебательных мод как симметричных, так и асимметричных комплексов: $v_{\mathrm{FF}-\mathrm{F}}$ - деформационное колебание по углу $\mathrm{F} \ldots \mathrm{F}^{-} \ldots \mathrm{F}, v_{\mathrm{Hb}, \mathrm{str}, \mathrm{s}}$ и $v_{\mathrm{Hb}, \mathrm{str}, \mathrm{a}}-$ симметричное и антисимметричное валентные колебания водородной связи, $v_{\mathrm{Hb}, \mathrm{in}, \mathrm{s}}$ и $v_{\mathrm{Hb}, \mathrm{in}, \mathrm{a}}-$ симметричное и антисимметричное деформационные колебания водородной связи в плоскости равновесной ядерной конфигурации, $v_{\mathrm{Hb} \text {,out,s }}$ и $v_{\mathrm{Hb}, \text { out }, \mathrm{a}}$ - симметричное и антисимметричное деформационные колебания водородной связи с выходом из плоскости равновесной ядерной конфигурации, $v_{\mathrm{L}-\mathrm{F}, \mathrm{s}}$ и $v_{\mathrm{L}-\mathrm{F}, \mathrm{a}}$ - симметричное и антисимметричное валентные колебания мономеров $\mathrm{L}_{1} \mathrm{~F}$ и $\mathrm{L}_{2} \mathrm{~F}$. Наряду с этими обозначениями в табл. 1 указаны и более простые обозначения $v_{i}(i=1-9)$, которые будут использованы ниже для компактного обозначения постоянных ангармонического взаимодействия $\chi_{i, j}$, а также обертонных и составных полос. Операцией симметрии, использованной в обозначениях колебательных мод, является отражение в плоскости, проходящей через ось симметрии $\left[\mathrm{F}(\mathrm{LF})_{2}\right]^{-}$ и перпендикулярной к плоскости равновесной конфигурации ядер. Эта операция является точной операцией симметрии для комплексов с $\mathrm{L}_{1}=\mathrm{L}_{2}$. Для остальных комплексов использование индексов $\mathrm{s}$ и а достаточно обосновано в случае мод $v_{\mathrm{Hb}, \mathrm{str}, \mathrm{s}}$ и $v_{\mathrm{Hb}, \mathrm{str}, \mathrm{a}}$, так как при- 
веденные массы двух водородных мостиков при $\mathrm{L}_{1} \neq \mathrm{L}_{2}$ остаются близкими. Использование индексов $\mathrm{s}$ и для деформационных колебаний водородных мостиков и валентных колебаний мономеров допустимо потому, что, хотя эти колебания происходят главным образом в одном мостике, они содержат примесь аналогичного колебания в другом мостике, происходящего в фазе или в противофазе. При этом всегда колебания $v_{\mathrm{Hb}, \mathrm{in,s}}, v_{\mathrm{Hb}, \text { out,a }}$, и $v_{\mathrm{L}-\mathrm{F}, \mathrm{s}}$ происходят в мостике, содержащем более легкую частицу L. Соответственно колебания $v_{\mathrm{Hb}, \mathrm{in}, \mathrm{a}}, v_{\mathrm{Hb}, \mathrm{out}, \mathrm{s}}$, и $v_{\mathrm{L}-\mathrm{F}, \mathrm{a}}$ локализованы в основном в мостике, содержащем более тяжелую частицу L. Деформационные и валентные колебания, локализованные преимущественно в мостике с более легкой частицей $\mathrm{L}$, всегда имеют более высокую частоту, чем соответствующие колебания другого водородного мостика.

Из табл. 1 видно, что частоты и интенсивности первых трех спектральных полос поглощения практически не меняются при изотопном замещении. Частоты остальных фундаментальных полос в симметричных комплексах $\left[\mathrm{F}(\mathrm{LF})_{2}\right]^{-}$закономерно уменьшаются с ростом массы L. Так же ведут себя интенсивности полос поглощения, за исключением $v_{\mathrm{Hb}, \mathrm{in}, \mathrm{a}}$ При изменении массы L в одном из мостиков, например, при переходе от $[\mathrm{FKaFKaF}]^{-}$к $\left[\mathrm{FKaFL}_{2} \mathrm{~F}\right]^{-}\left(\mathrm{L}_{2}=\mathrm{H}, \mathrm{D}, \mathrm{T}\right)$ частоты $v_{\mathrm{Hb}, \mathrm{in}, \mathrm{s}}, \nu_{\mathrm{Hb}, \mathrm{out}, \mathrm{a}}$ и $v_{\mathrm{L}-\mathrm{F}, \mathrm{s}}$ меняются незначительно, так как в асимметричных комплексах эти колебания связаны в основном с более легким мономером. Но интенсивности этих трех полос резко меняются при переходе от $[\mathrm{FKaFKaF}]^{-}$к $[\mathrm{FKaFHF}]^{-}$, а далее при переходе к $[\mathrm{FKaFDF}]^{-}$и $[\mathrm{FKaFTF}]^{-}$они меняются незначительно, за исключением полосы $v_{\mathrm{Hb}, \mathrm{in,s}}$. Из разложений нормальных координат по смещениям декартовых координат ядер следует, что в комплексе $[\mathrm{FKaFTF}]^{-}$ нормальное колебание $v_{\mathrm{Hb} \text {,in,s }}$ содержит значительную примесь валентного колебания $\mathrm{T}-\mathrm{F}$, так как частоты этих колебаний близки. В свою очередь, нормальное колебание $v_{\mathrm{L}-\mathrm{F}, \mathrm{a}}$ содержит примесь плоского деформационного колебания F... Ka-F. В комплексе $[\mathrm{FKaFDF}]^{-}$ такое смешивание тоже происходит, но с другой относительной фазой и с меньшей силой. В результате полоса $v_{\mathrm{Hb}, \mathrm{in}, \mathrm{s}}$ несколько понижает свою интенсивность в $[\mathrm{FKaFDF}]^{-}$, а в $[\mathrm{FKaFTF}]^{-}$она резко увеличивает интенсивность за счет полосы валентного колебания T-F $\left(v_{\mathrm{L}-\mathrm{F}, \mathrm{a})}\right.$. Подчеркнем, что эти взаимодействия колебательных степеней свободы проявляются уже на этапе гармонического расчета. Аналогичное поведение частот и интенсивностей полос поглощения $v_{\mathrm{Hb}, \mathrm{in}, \mathrm{s}}, v_{\mathrm{Hb} \text {,out,a }}$ и $v_{\mathrm{L}-\mathrm{F}, \mathrm{s}}$ наблюдается при переходе от $[\mathrm{FHFHF}]^{-}$к $[\mathrm{FHFDF}]^{-}$ и $[\mathrm{FHFTF}]^{-}$, а также при переходе от $[\mathrm{FDFDF}]^{-}$к $[\mathrm{FDFTF}]^{-}$, но в этих случаях нет заметного смешивания деформационных и валентных колебаний. Что касается аналогичных закономерностей изменения частот и интенсивностей полос $v_{\mathrm{Hb} \text {,in,a }}, v_{\mathrm{Hb} \text {,out,s }}$ и $v_{\mathrm{L}-\mathrm{F}, \mathrm{a}}$, то они видны при сравнении спектральных параметров симметричного комплекса $\left[\mathrm{F}(\mathrm{LF})_{2}\right]^{-}$с параметрами асимметричного комплекса, в котором частица $\mathrm{L}$ является более тяжелой, например, при сравнении параметров [FDFDF $]^{-}$ с параметрами $[\mathrm{FKaFDF}]^{-}$и $[\mathrm{FHFDF}]^{-}$. Обнаруженные различия в поведении частот и интенсивностей фундаментальных полос поглощения при переходе от одного изотополога к другому показывают, что интенсивности более чувствительны, чем частоты к изменению взаимодействия между колебаниями водородных мостиков, вызываемому изотопным замещением.

\section{Расчеты ангармонических постоянных}

Потенциальные поверхности рассматриваемых нами изотопологов должны совпадать, если их рассчитывать в адиабатическом приближении в пространстве декартовых смещений ядер. Но нормальные координаты, в пространстве которых выполняются ангармонические расчеты частот и интенсивностей, зависят от масс ядер и, следовательно, изменяются при переходе от одного изотополога к другому. Поэтому численные значения ангармонических параметров комплексов $\left[\mathrm{FL}_{1} \mathrm{FL}_{2} \mathrm{~F}\right]^{-}$ могут меняться при изотопном замещении. В теории возмущений [16] используется следующее выражение для энергии колебательных уровней $\varepsilon_{n}$ :

$$
\begin{aligned}
\varepsilon_{n} & =\chi_{0}+\sum_{i=1}^{N} \omega_{i}\left(n_{i}+\frac{1}{2}\right) \\
& +\sum_{i=1}^{N} \sum_{j=1}^{N} \chi_{i j}\left(n_{i}+\frac{1}{2}\right)\left(n_{j}+\frac{1}{2}\right) .
\end{aligned}
$$

Здесь $n$ - совокупность квантовых чисел рассматриваемого колебательного состояния, $\chi_{0}$ - энергия нулевых колебаний системы, $\omega_{i}$ - гармоническая частота нормального колебания $i, \chi_{i, j}-$ постоянная ангармонического взаимодействия колебаний $i$ и $j$. В свою очередь, постоянные $\chi_{i, j}$ выражаются через производные от потенциальной энергии по нормальным координатам, частоты $\omega_{i}$ и постоянные кориолисова взаимодействия. Поэтому интересно увидеть, как изменяются численные значения ангармонических постоянных $\chi_{i, j}$ при переходе от одного комплекса $\left[\mathrm{FL}_{1} \mathrm{FL}_{2} \mathrm{~F}\right]^{-}$к другому.

В табл. 2 показаны значения, вычисленные при помощи программы Gaussian 16 [14], постоянных $\chi_{i, j}$, дающих главные вклады в энергии $\varepsilon_{n}$. Смысл индексов $i$ и $j$ пояснен в первом столбце табл. 1. В работе [17] отмечалось, что применение теория возмущений к расчету характеристик, связанных с низкочастотными модами, может давать ошибочные результаты, так как величины возмущений могут быть больше частот этих мод. По этой причине в табл. 2 не указываются постоянные, содержащие индекс колебания $v_{1}$. Приведены только те постоянные $\chi_{i, j}$, значения которых превышают $10 \mathrm{~cm}^{-1}$ хотя бы для одного из десяти рассмотренных изотопологов. 
Таблица 2. Значения (в $\left.\mathrm{cm}^{-1}\right)$ ангармонических постоянных $\chi_{i, j}$

\begin{tabular}{|c|c|c|c|c|c|c|c|c|c|c|}
\hline$i, j$ & {$[\mathrm{FKaFKaF}]^{-}$} & {$[\mathrm{FHFHF}]^{-}$} & {$[\mathrm{FDFDF}]^{-}$} & {$[\text {FTFTF }]^{-}$} & {$[\mathrm{FKaFHF}]^{-}$} & {$[\mathrm{FKaFDF}]^{-}$} & {$[\mathrm{FKaFTF}]^{-}$} & {$[\mathrm{FHFDF}]^{-}$} & {$[\text {FHFTF }]^{-}$} & {$[\mathrm{FDFTF}]^{-}$} \\
\hline 2,4 & -23.5 & -16.5 & -11.3 & -8.4 & -15.8 & -11.8 & -5.3 & -11.7 & -9.6 & -8.0 \\
\hline 2,8 & 43.8 & 30.4 & 21.5 & 17.3 & 46.0 & 39.5 & 34.1 & 27.1 & 26.1 & 22.9 \\
\hline 2,9 & 50.3 & 35.4 & 23.9 & 18.8 & 34.1 & 24.7 & 16.5 & 26.7 & 22.4 & 17.5 \\
\hline 3,7 & -16.9 & -11.8 & -8.2 & -5.9 & -13.1 & -14.7 & -16.1 & -10.9 & -10.7 & -6.7 \\
\hline 3,8 & 67.7 & 44.2 & -39.4 & -22.1 & 38.4 & 40.8 & 46.4 & 35.9 & 29.2 & 15.6 \\
\hline 3,9 & 0.9 & 0.6 & 66.2 & 40.3 & 18.3 & 8.8 & 3.7 & 2.1 & 4.9 & 7.2 \\
\hline 4,4 & -69.9 & -33.6 & -16.3 & -10.7 & -146.2 & -151.7 & -129.1 & -71.8 & -73.1 & -29.8 \\
\hline 4,6 & -20.3 & -9.2 & -3.7 & -2.3 & 12.3 & 9.5 & 16.5 & 6.1 & 5.1 & 3.2 \\
\hline 4,7 & -13.8 & -7.0 & -3.8 & -2.3 & -47.7 & -47.6 & -49.7 & -22.5 & -22.7 & -10.3 \\
\hline 4,8 & 201.2 & 98.1 & 47.8 & 31.4 & 586.8 & 604.2 & 596.5 & 290.9 & 287.7 & 116.6 \\
\hline 4,9 & -0.4 & -1.8 & -0.7 & 0.1 & -86.8 & -69.3 & -170.5 & -45.2 & -29.2 & -16.9 \\
\hline 5,5 & -45.6 & -22.1 & -10.6 & -6.8 & -88.6 & -37.5 & -4.2 & -37.1 & -23.2 & -2.1 \\
\hline 5,8 & 92.0 & 44.8 & 21.9 & 14.4 & -73.5 & -52.7 & -41.8 & -36.2 & -29.4 & -17.1 \\
\hline 5,9 & -43.5 & -21.9 & -10.9 & -7.0 & 345.9 & 148.7 & 7.8 & 142.8 & 92.9 & 4.7 \\
\hline 6,6 & -36.5 & -19.1 & -9.1 & -5.9 & -82.5 & -35.0 & -22.77 & -35.5 & -22.1 & -23.0 \\
\hline 6,7 & -21.5 & -10.0 & -2.2 & -0.2 & 17.8 & 12.0 & 10.6 & 8.5 & 7.5 & 5.1 \\
\hline 6,8 & 102.8 & 50.7 & 26.2 & 14.3 & -80.8 & -59.3 & -49.5 & -41.1 & -34.9 & -21.9 \\
\hline 6,9 & -29.3 & -10.4 & -11.1 & -6.6 & 317.5 & 135.3 & 79.6 & 136.0 & 85.9 & 86.4 \\
\hline 7,7 & 52.4 & -30.0 & -12.3 & -8.6 & -133.3 & -145.6 & -147.7 & -64.8 & -67.4 & -27.7 \\
\hline 7,8 & 124.2 & 58.5 & 25.7 & 18.9 & 520.1 & 559.4 & 567.1 & 255.7 & 259.9 & 102.9 \\
\hline 7,9 & -38.7 & -0.9 & -9.8 & -7.7 & -96.5 & -61.8 & -48.6 & -50.1 & -36.9 & -22.0 \\
\hline 8,8 & -162.9 & -83.7 & -44.7 & -31.6 & -602.6 & -643.6 & -649.9 & -304.9 & -321.7 & -139.2 \\
\hline 8,9 & -1188.2 & -599.0 & -374.9 & -253.1 & 224.2 & 212.7 & 262.0 & 114.4 & 121.9 & 14.4 \\
\hline 9,9 & -234.1 & -119.1 & -62.3 & -43.0 & -312.8 & -162.6 & -93.3 & -161.2 & -116.8 & -100.9 \\
\hline
\end{tabular}

Прежде всего из табл. 2 видно, что абсолютные значения постоянных $\chi_{i, j}$ могут быть очень велики. Например, $\chi_{8,9}=-1188.2 \mathrm{~cm}^{-1}$ в случае $[\mathrm{FKaFKaF}]^{-}$. Для комплексов, которые наблюдаются в реальных экспериментах, максимальные по модулю значения имеют константы $\chi_{8,9}=-599.0 \mathrm{~cm}^{-1}\left([\mathrm{FHFHF}]^{-}\right)$и $-374.9 \mathrm{~cm}^{-1}$ $\left([\mathrm{FDFDF}]^{-}\right)$и $\chi_{8,8}=-304.9 \mathrm{~cm}^{-1}\left([\mathrm{FHFDF}]^{-}\right)$. Почти все приведенные в табл. 2 постоянные монотонно уменьшаются по модулю, но сохраняют знак при переходе от $[\mathrm{FHFHF}]^{-}$к $[\mathrm{FTFTF}]^{-}$. Исключением являются $\chi_{3,8}$ и $\chi_{3,9}$. Тот факт, что значения $\chi_{3,8}$ и $\chi_{3,9}$ резко меняются при переходе от $[\mathrm{FHFHF}]^{-}$к $[\mathrm{FDFDF}]^{-}$, может быть связан с тем, что колебания по $R(\mathrm{~L}-\mathrm{F})$ и $R(\mathrm{~F}-\mathrm{F})$ практически независимы в $[\mathrm{FKaFKaF}]^{-}$и $[\mathrm{FHFHF}]^{-}$), но довольно сильно смешиваются в нормальных колебаниях $[\mathrm{FDFDF}]^{-}$и $[\mathrm{FTFTF}]^{-}$. Отличия значений $\chi_{i, j}$ для асим- метричных комплексов $\mathrm{FL}_{1} \mathrm{FL}_{2} \mathrm{~F}^{-}$от соответствующих значений для симметричных комплексов возникают потому, что, за исключением мод $v_{1}-v_{3}$, колебания асимметричных комплексов достаточно строго локализованы в одном из водородных мостиков. Как правило, значения $\chi_{i, j}$ для $\left[\mathrm{FL}_{1} \mathrm{FL}_{2} \mathrm{~F}\right]^{-}$уменьшаются с ростом масс $\mathrm{L}_{1}$ и $\mathrm{L}_{2}$ и, следовательно, с уменьшением частот и амплитуд колебаний. Из общей тенденции выпадает значение $\chi_{4,9}$ в $[\mathrm{FKaFTF}]^{-}$. Этот факт объясняется обсуждаемым в предыдущем разделе смешиванием плоского деформационного колебания $\mathrm{F}^{-}$. . Ka-F и валентного колебания $\mathrm{T}-\mathrm{F}$ в этом изотопологе. Данные табл. 2 подтверждают корреляцию по свойствам симметрии между колебаниями симметричных и асимметричных комплексов. Наличие симметричности в валентном колебании $v_{8}$ более легкого мономера в $\left[\mathrm{FL}_{1} \mathrm{FL}_{2} \mathrm{~F}\right]^{-}$согласуется с тем фак- 
Таблица 3. Ангармонические значения частот $v\left(\mathrm{~cm}^{-1}\right)$ и интенсивностей $S\left(\mathrm{~km} \cdot \mathrm{mol}^{-1}\right)$ ИК полос поглощения изотопологов комплекса $\left[\mathrm{F}(\mathrm{HF})_{2}\right]^{-}$

\begin{tabular}{|c|c|c|c|c|c|c|c|c|c|c|}
\hline \multirow{2}{*}{ Полоса } & \multicolumn{10}{|c|}{$v(S)$} \\
\hline & {$[\mathrm{FKaFKaF}]^{-}$} & {$[\mathrm{FHFHF}]^{-}$} & {$[\mathrm{FDFDF}]^{-}$} & {$\left[\right.$FTFTF $^{-}$} & {$[\mathrm{FKaFHF}]^{-}$} & {$[\mathrm{FKaFDF}]^{-}$} & {$[\mathrm{FKaFTF}]^{-}$} & {$[\mathrm{FHFDF}]^{-}$} & {$[\text {FHFTF }]^{-}$} & {$[\mathrm{FDFTF}]^{-}$} \\
\hline$v_{1}=v_{\mathrm{FF}-\mathrm{F}}$ & $45(0.2)$ & $46(0.1)$ & $46(0.08)$ & $46(0.08)$ & $46(0.13)$ & $47(0.13)$ & $47(0.14)$ & $46(0.1)$ & $46(0.11)$ & $46(0.08)$ \\
\hline$v_{2}=v_{\mathrm{Hb}, \mathrm{str}, \mathrm{s}}$ & $357(8)$ & $347,(8)$ & & $326(9)$ & $352(9)$ & $345(14)$ & $339(18)$ & & & $330(7)$ \\
\hline$v_{3}=v_{\mathrm{Hb}, \mathrm{str}, \mathrm{a}}$ & $435(293)$ & $426(272)$ & $414(258)$ & $404(251)$ & $430(282)$ & $425(271)$ & $421(262)$ & $420(264)$ & $415(238)$ & $409(254)$ \\
\hline$v_{4}=v_{\mathrm{Hb}, \mathrm{in}, \mathrm{s}}$ & $1613(216)$ & $1159(140)$ & $844(75)$ & $703(72)$ & $1580(144)$ & $1590(175)$ & $1623(948)$ & $1133(82)$ & $1135(78)$ & $824(64)$ \\
\hline$v_{5}=v_{\mathrm{Hb}, \mathrm{in}, \mathrm{a}}$ & $1514(14)$ & $1092(14)$ & $792(21)$ & $658(20)$ & $1119(67)$ & $808(48)$ & $672(39)$ & $812(45)$ & $676(37)$ & $677(35)$ \\
\hline$v_{6}=v_{\mathrm{Hb}, \text { out }, \mathrm{s}}$ & $1546(185)$ & $1109(203)$ & $805(103)$ & $671(145)$ & $1104(106)$ & $795(60)$ & $660(45)$ & $801(60)$ & $665(40)$ & $667(43)$ \\
\hline$v_{7}=v_{\mathrm{Hb}, \text { out }, \mathrm{a}}$ & $1535(190)$ & $1109(3)$ & $802(17)$ & $665(11)$ & $1551(196)$ & $1558(195)$ & $1562(196)$ & $1113(104)$ & $1116(105)$ & $805(62)$ \\
\hline$\nu_{8}=v_{\mathrm{L}-\mathrm{F}, \mathrm{s}}$ & $3372(453)$ & $2590(230)$ & $1801(186)$ & $1547(157)$ & $3144(289)$ & $3061(230)$ & $3106(174)$ & $2364(562)$ & $2339(596)$ & $1751(399)$ \\
\hline$\nu_{9}=\nu_{\mathrm{L}-\mathrm{F}, \mathrm{a}}$ & 2093 (4869) & $1747(2430)$ & 1381 (1195) & 1199 (659) & $2342(834)$ & $1776(904)$ & $1494(175)$ & $1738(962)$ & $1494(751)$ & $1456(781)$ \\
\hline $2 v_{4}$ & 3144 (298) & $2270(46)$ & $1667(20)$ & $1391(7)$ & $3005(43)$ & $3006(33)$ & 3045 (126) & $2197(22)$ & $2198(22)$ & $1602(21)$ \\
\hline $2 v_{5}$ & $2990(219)$ & $2172(153)$ & $1535(62)$ & $1287(28)$ & $2153(27)$ & $1568(13)$ & $1316(9)$ & $1590(15)$ & $1331(14)$ & $1314(39)$ \\
\hline $2 v_{6}$ & $3051(596)$ & 2204 (337) & $1599(4)$ & $1333(5)$ & $1950(3456)$ & $1483(772)$ & $1250(272)$ & $1484(928)$ & $1261(373)$ & $1259(467)$ \\
\hline $2 v_{7}$ & $2823(613)$ & 2084 (199) & $1591(4)$ & $1324(6)$ & $2698(3317)$ & $2677(4776)$ & $2714(4039)$ & $2033(1027)$ & $2022(1440)$ & $1568(97)$ \\
\hline$v_{2}+v_{8}$ & 3697 (3) & $2860(1)$ & 2157 (1) & $1890(1)$ & $3541(22)$ & $3471(16)$ & $3479(8)$ & $2728(4)$ & $2700(4)$ & $2142(2)$ \\
\hline$v_{2}+v_{9}$ & $2763(160)$ & $2318(56)$ & $1887(17)$ & $1681(10)$ & $2728(16)$ & $2145(6)$ & $1828(5)$ & $2108(8)$ & $1852(4)$ & $1775(39)$ \\
\hline$v_{3}+v_{8}$ & $3799(235)$ & $2953(86)$ & $2175(30)$ & $1929(18)$ & $3612(593)$ & $3552(722)$ & $3574(786)$ & $2814(163)$ & 2784 (188) & $2214(42)$ \\
\hline$v_{3}+v_{9}$ & $2715(108)$ & $2255(159)$ & $2008(68)$ & $1781(23)$ & 2791 (439) & $2209(94)$ & $1898(37)$ & $2164(201)$ & $1915(64)$ & $1882(58)$ \\
\hline$v_{4}+v_{5}$ & $3354(1359)$ & $2419(971)$ & $1766(768)$ & $1482(657)$ & $2710(31)$ & $2394(0.3)$ & $2284(0.2)$ & $1950(29)$ & $1813(0.3)$ & $1507(138)$ \\
\hline$v_{6}+v_{7}$ & $3087(13)$ & $2227(13)$ & $1618(34)$ & $1346(11)$ & $2672(9)$ & $2365(0.1)$ & $2233(0.02)$ & $1923(6)$ & $1788(0.2)$ & $1477(0.6)$ \\
\hline
\end{tabular}

Примечание. Результаты расчетов $[\mathrm{FHFHF}]^{-}$и $[\mathrm{FDFDF}]^{-}$взяты из [13].

том, что для всех асимметричных комплексов $v_{8,8}$ значительно больше по абсолютной величине, чем $v_{9,9}$. В свою очередь, наличие антисимметрии валентного колебания $v_{9}$ более тяжелого мономера подтверждается, например, тем, что $v_{2,9}=34.1 \mathrm{~cm}^{-1}$ для $[\mathrm{FKaFHF}]^{-}$близко значению $35.4 \mathrm{~cm}^{-1}$ для $[\mathrm{FHFHF}]^{-}$, а $\chi_{2,9}=24.7 \mathrm{~cm}^{-1}$ для $[\mathrm{FKaFDF}]^{-}$близко значению $23.9 \mathrm{~cm}^{-1}$ для [FDFDF $]^{-}$.

\section{Ангармонические расчеты частот и интенсивностей ИК полос поглощения}

В табл. 3 приведены ангармонические значения частот и интенсивностей всех фундаментальных полос комплекса $[\mathrm{FHFHF}]^{-}$и его изотопологов, а также частоты и интенсивности ряда обертонных и составных полос. Различия между гармоническими и ангармоническими значениями частот и интенсивностей фундаментальных полос $v_{1}, v_{2}$ и $v_{3}$ всех десяти изотопологов можно считать незначительными. При учете ангармонических эффектов частоты остальных фундаментальных полос рассмотренных комплексов уменьшаются, но значения интенсивностей в некоторых случаях увеличиваются. Высокочастотные полосы $v_{4}-v_{9}$ можно разделить на две группы по влиянию ангармонических эффектов на спектральные параметры. Ангармонические значения частот полос поглощения $v_{4}-v_{7}$ ниже гармонических значений в среднем на $4 \%$. Понижение значений интенсивностей этой группы полос, как правило, более заметное (от 5 до 25\%). Но в случае полосы $v_{4}$ в $[\mathrm{FKaFDF}]^{-}$ и $[\mathrm{FKaFTF}]^{-}$, полосы $v_{6}$ в $[\mathrm{FTFTF}]^{-}$и полосы $v_{7}$ в $[\mathrm{FKaFKaF}]^{-}$значения интенсивности сильно увеличиваются при учете ангармонических поправок. В группе полос $v_{8}$ и $v_{9}$, связанных с внутренними валентными колебаниями мономеров, влияние ангармоничности на частоты и интенсивности более сильное. Для симметричных комплексов с $\mathrm{L}_{1}=\mathrm{L}_{2}$ наблюдается понижение частоты симметричного колебания $v_{8}$ от 7 до 17\% при учете ангармонических эффектов, а интенсивность этой полосы падает в 1.5-3 раза. Для этих же комплексов 
частота антисимметричного колебания $v_{9}$ понижается на $22-40 \%$, а интенсивность полосы $v_{9}$ падает в два раза. Для асимметричных комплексов с $\mathrm{L} \neq \mathrm{L}_{2}$ частоты колебаний $v_{8}$ и $v_{9}$ понижаются на 6-15\%, т. е. почти так же, как и в симметричных комплексах. Но интенсивности полос $v_{8}$ и $v_{9}$ понижаются драматически: от двух до двадцати раз в случае $v_{8}$ и от полутора до четырех раз в случае $v_{9}$.

Значительное понижение интенсивности полос валентных колебаний мономеров в асимметричных комплексах приводит к следующему поразительному результату. Вспомним, что гармонический расчет симметричных комплексов дает более высокую частоту и более низкую интенсивность для симметричного колебания $v_{8}=v_{\mathrm{L}-\mathrm{F}, \mathrm{s}}$, чем для антисимметричного колебания $v_{9}=v_{\mathrm{L}-\mathrm{F}, \mathrm{a}}$. Гармонический расчет асимметричных комплексов предсказывает, что валентное колебание более легкого мономера сохраняет следы симметричного колебания и имеет более высокую частоту, чем аналогичное колебание другого мономера в комплексе. Но во всех асимметричных комплексах, кроме $[\mathrm{FDFTF}]^{-}$, валентное колебание более легкого мономера имеет более высокую гармоническую интенсивность. При учете ангармоничности интенсивность полос $v_{8}$ понижается сильнее, чем интенсивность полос $v_{9}$. В результате интенсивность полосы $v_{8}$ оказывается ниже интенсивности $v_{9}$ и в асимметричных комплексах. Таким образом, в асимметричных комплексах $\left[\mathrm{FL}_{1} \mathrm{FL}_{2} \mathrm{~F}\right]^{-}$полоса поглощения $v_{9}$, связанная с валентным колебанием более тяжелого мономера, должна иметь более высокую интенсивность. Этот вывод показывает ошибочность предположений, сделанных в [6] при анализе спектра $[\text { FHFDF }]^{-}$, о том, что полоса поглощения, наблюдаемая в эксперименте на частоте $1707 \mathrm{~cm}^{-1}$, связана с колебанием $\mathrm{H}-\mathrm{F}$, а полоса D-F должна быть слабее и должна лежать при более низких частотах. Совпадение полученного нами ангармонического значения частоты $1738 \mathrm{~cm}^{-1}$ для колебания D-F в изолированном комплексе (табл. 3) со значением $1707 \mathrm{~cm}^{-1}$ в матричном спектре следует признать очень хорошим. Для полосы колебания $\mathrm{H}-\mathrm{F}$ в $[\mathrm{FHFDF}]^{-}$наш ангармонический расчет предсказывает частоту $2364 \mathrm{~cm}^{-1}$ и интенсивность $562 \mathrm{~km} \cdot \mathrm{mol}^{-1}$. Вероятно, эта полоса не была замечена в [6], потому что она попадает в область очень сильного поглощения примеси $\mathrm{CO}_{2}$. В четырехмерном вариационном расчете $[11]$ комплекса $[\mathrm{FHFDF}]^{-}$были получены достаточно точные значения частот колебаний $\mathrm{D}-\mathrm{F}$ и $\mathrm{H}-\mathrm{F}$ (1697 и $2294 \mathrm{~cm}^{-1}$ ), а значения соответствующих интенсивностей $\left(1883\right.$ и $\left.1483 \mathrm{~km} \cdot \mathrm{mol}^{-1}\right)$ указывают на более высокую интенсивность полосы колебания D-F, как и ангармонические расчеты по теории возмущений. Тот факт, что вариационные значения интенсивностей приблизительно в два раза выше ангармонических значений, свидетельствует о необходимости учета ангармонических взаимодействий между всеми модами при расчете интенсивностей. Стоит отметить, что в комплексе $[\mathrm{FKaFTF}]^{-}$ангармонические значения интенсивностей $v_{8}$ и $v_{9}$ почти совпадают из-за обсуждаемого вы- ше сильного смешивания валентного колебания $\mathrm{T}-\mathrm{F}$ и плоского деформационного колебания F. . Ка-F. В результате полоса $v_{9}$ отдает часть своей интенсивности, а полоса $v_{4}$ становится самой сильной фундаментальной полосой $[\mathrm{FKaFTF}]^{-}$.

В табл. 3 приведены спектральные параметры наиболее интенсивных обертонных и составных полос, попадающих в область поглощения полос валентных колебаний мономеров L-F каждого комплекса. К сожалению, в настоящее время нет экспериментальных данных об обертонах и составных полосах обсуждаемых нами комплексов. Из табл. 3 видно, что обертонные полосы поглощения могут иметь значительную интенсивность для $[\mathrm{FKaFKaF}]^{-}$и $[\mathrm{FHFHF}]^{-}$, но их интенсивность низка в случае более тяжелых симметричных комплексов. Что касается обертонов асимметричных комплексов, то очень высокие значения интенсивности обертонов полос неплоских деформационных колебаний, полученные по теории возмущений, по-видимому, нуждаются в проверке независимым вариационным расчетом. Высокие значения интенсивности некоторых составных полос не вызывают сомнений, так как четырех- и шестимерные вариационные расчеты [12,13] предсказывали ранее возможность сильных резонансов между возбужденными состояниями валентных колебаний мономеров и составными возбужденными состояниями деформационных колебаний. При наличии таких резонансов происходит значительный перенос интенсивности от полос валентных мод к полосам обертонов и составных колебаний деформационных мод.

\section{Заключение}

В настоящей работе были выполнены гармонические и ангармонические расчеты частот и интенсивностей ИК полос поглощения комплексов $\left[\mathrm{F}(\mathrm{KaF})_{2}\right]^{-},\left[\mathrm{F}(\mathrm{TF})_{2}\right]^{-}$, $[\mathrm{FKaFHF}]^{-}, \quad[\mathrm{FKaFDF}]^{-}, \quad[\mathrm{FKaFTF}]^{-}, \quad[\mathrm{FHFDF}]^{-}$,

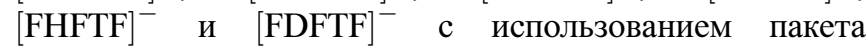
программ Gaussian 16 [14] в приближении МР2/6$311++\mathrm{G}(3 d f, 3 p d) \quad$ с учетом ошибки наложения базисных наборов мономеров. Ангармонические расчеты выполнены по методу теории возмущений второго порядка $[15,16]$. Полученные результаты вместе с результатами предыдущего расчета [13] тем же методом комплексов $\left[\mathrm{F}(\mathrm{HF})_{2}\right]^{-}$и $\left[\mathrm{F}(\mathrm{DF})_{2}\right]^{-}$проанализированы c целью определения тенденций в изменениях спектральных параметров при переходе от одного симметричного изотополога к другому и при переходе от симметричных изотопологов к асимметричным. Результаты выполненных в данной работе девятимерных ангармонических расчетов сравниваются с результатами четырех- и шестимерных вариационных расчетов [9-13].

Было показано, что полосы симметричных валентных колебаний мономеров в комплексах $\left[\mathrm{F}(\mathrm{LF})_{2}\right]^{-}$ $(\mathrm{L}=\mathrm{Ka}, \mathrm{H}, \mathrm{D}, \mathrm{T})$ имеют более высокую частоту и меньшую интенсивность, чем полосы антисимметричных 
колебаний. Частоты фундаментальных полос симметричных комплексов уменьшаются с ростом массы L. Аналогично ведут себя интенсивности этих полос, за исключением полос валентных колебаний водородных связей и антисимметричных деформационных колебаний. Анализ констант ангармонических взаимодействий показал, что они уменьшаются по абсолютной величине с ростом массы частиц $\mathrm{L}$ и имеют наибольшие значения в случае валентных колебаний мономеров и взаимодействия этих колебаний с деформационными колебаниями. При замене в одном из мостиков симметричного комплекса $\left[\mathrm{F}\left(\mathrm{L}_{i} \mathrm{~F}\right)_{2}\right]^{-}$частицы $\mathrm{L}_{i}$ на частицу $\mathrm{L}_{j}$ с другой массой каждое из валентных колебаний $\mathrm{L}_{i}-\mathrm{F}$ и $\mathrm{L}_{j}-\mathrm{F}$ в полученном асимметричном комплексе локализуется в своем мостике, но сохраняет примесь колебания другого мономера, а их частоты близки полусумме частот симметричного и антисимметричного колебаний соответствующих симметричных комплексов. В гармонических расчетах всех асимметричных комплексов, кроме $[\mathrm{FDFTF}]^{-}$, наиболее интенсивной оказывается полоса поглощения по валентному колебанию более легкого мономера, частота колебания которого выше частоты колебания в другом мономере. Но в ангармонических расчетах полоса валентного колебания более тяжелого мономера оказывается более интенсивной во всех асимметричных комплексах. За исключением $[\mathrm{FKaFTF}]^{-}$, полоса антисимметричного валентного колебания $\mathrm{L}-\mathrm{F}$ является самой сильной из всех фундаментальных полос. $\mathrm{B}[\mathrm{FKaFTF}]^{-}$полоса плоского деформационного колебания $v_{\mathrm{Hb}, \mathrm{in,s}}$ является наиболее интенсивной благодаря взаимодействию этого колебания с валентным колебанием T-F. Предсказанное ангармоническим расчетом значение $1738 \mathrm{~cm}^{-1}$ для колебания $\mathrm{D}-\mathrm{F}$ в изолированном комплексе $[\mathrm{FHFDF}]^{-}$находится в хорошем согласии со значением $1707 \mathrm{~cm}^{-1}$, полученным в матричном эксперименте [6] и ошибочно приписанным там колебанию $\mathrm{H}-\mathrm{F}$. Для колебания $\mathrm{H}-\mathrm{F}$ этого комплекса настоящий расчет предсказывает частоту $2364 \mathrm{~cm}^{-1}$. Возможно, полоса $\mathrm{H}-\mathrm{F}$ не была обнаружена в [6] потому, что она попадает в область очень сильного поглощения примеси $\mathrm{CO}_{2}$. Высокие теоретические значения интенсивности некоторых обертонных и составных полос поглощения свидетельствуют о возможности резонансов и переноса интенсивности между спектральными переходами.

\section{Финансирование работы}

Работа выполнена при финансовой поддержке Российского фонда фундаментальных исследований, грант № 18-03-00520.

\section{Конфликт интересов}

Авторы заявляют, что у них нет конфликта интересов.

\section{Список литературы}

[1] Forrester J.D., Senter M.E., Zalkin A., Templeton D. // Acta Cryst. 1963. V. 16. P. 58.

[2] Azman A., Ochvirk A., Hadzi D., Giguere P., Schneider M. // Can. J. Chem. 1967. V. 45. P. 1347.

[3] Harmon K.M., Gennick I. // J. Mol. Struct. 1977. V. 38. P. 97.

[4] Gennick I., Harmon K.M., Potvin M.M. // Inorg. Chem. 1977. V. 16. P. 2033.

[5] Lovelace R.R., Harmon K.M. // J. Mol. Struct. 1989. V. 193. P. 247.

[6] Hunt R.D., Andrews L. // J. Chem. Phys. 1987. V. 87. P. 6819.

[7] Chandler W.D., Johnson K.E., Campbell J.L.E. // Inorg. Chem. 1995. V. 34. P. 4943.

[8] Shodai Y., Kohara S., Ohishi Y., Inaba M., Tasaka A. // J. Phys. Chem. A. 2004. V. 108. P. 1127.

[9] Bulychev V.P., Buturlimova M.V. // J. Mol. Struct. 2009. V. 928 . P. 32.

[10] Булычев В.П., Бутурлимова М.В., Тохадзе К.Г. // Опт. и спектр. 2010. Т. 108. С. 931.

[11] Bulychev V.P., Buturlimova M.V., Tokhadze K.G. // Phys. Chem. Chem. Phys. 2011. V. 13. P. 14019.

[12] Булычев В.П., Бутурлимова М.В., Тохадзе К.Г. // Опт. и спектр. 2012. Т. 113. С. 653.

[13] Bulychev V.P., Buturlimova M.V., Tokhadze K.G. // J. Chem. Phys. 2018. V. 149. P. 104306.

[14] Gaussian 16, Revision A.03.Frisch M.J., Trucks G.W., Schlegel H.B., Scuseria G.E., Robb M.A., Cheeseman J.R., Scalmani G., Barone V., Petersson G.A., Nakatsuji H., Li X., Caricato M., Marenich V., Bloino J., Janesko B.G., Gomperts R., Mennucci B., Hratchian H.P., Ortiz J.V., Izmaylov A.F., Sonnenberg J.L., Williams-Young D., Ding F., Lipparini F., Egidi F., Goings J., Peng B., Petrone A., Henderson T., Ranasinghe D., Zakrzewski V.G., Gao J., Rega N., Zheng G., Liang W., Hada M., Ehara M., Toyota K., Fukuda R., Hasegawa J., Ishida M., Nakajima T., Honda Y., Kitao O., Nakai H., Vreven T., Throssell K., Montgomery J.A., Jr., Peralta J.E., Ogliaro F., Bearpark M.J., Heyd J.J., Brothers E.N., Kudin K.N., Staroverov V.N., Keith T.A., Kobayashi R., Normand J., Raghavachari K., Rendell A.P., Burant J.C., Iyengar S.S., Tomasi J., Cossi M., Millam J.M., Klene M., Adamo C., Cammi R., Ochterski J.W., Martin R.L., Morokuma K., Farkas O., Foresman J.B., Fox D.J. Gaussian, Inc., Wallingford CT, 2016.

[15] Barone V. // J. Chem. Phys. 2005. V. 122. P. 014108.

[16] Bloino J. // J. Phys. Chem. A. 2015. V. 119. P. 5269.

[17] Булычев В.П., Енгалычева Е.А., Тохадзе К.Г. // Опт. и спектр. 2019. Т. 126. С. 404. 